ESAIM: PROCEEDINGS, August 2009, Vol. 28, p. 195-210

M. Ismail, B. Maury \& J.-F. Gerbeau, Editors

\title{
FLUID-PARTICLES FLOWS: A THIN SPRAY MODEL WITH ENERGY EXCHANGES
}

\author{
Laurent Boudin $^{1,2}$, Benjamin Boutin ${ }^{1,3}$, Bruno Fornet ${ }^{4}$, Thierry Goudon ${ }^{5,6}$, \\ Pauline Lafitte ${ }^{5,6}$, Frédéric Lagoutière ${ }^{5,7}$ and Benoît Merlet ${ }^{8}$
}

\begin{abstract}
This paper is devoted to an asymptotic analysis of a fluid-particles coupled model, in the bubbling regime. On the theoretical point of view, we extend the analysis done in [4] for the case of an isentropic gas to the case of an ideal gas, thus adding the internal energy, or temperature, which is unknown. We formally derive the bubbling limit system in the same way as in [4] and propose a numerical scheme to solve this limit system.

The numerical resolution of the non-limit system, and the numerical analysis of the asymptotic properties of the scheme (e.g. the asymptotic preserving property), as performed in [4], is at study.

Résumé. Nous proposons ici une analyse asymptotique formelle d'un modèle de couplage entre une densité de particules et un fluide, dans la limite dite bubbling. Cette analyse est effectuée en suivant les pas de [4] où le fluide considéré est isentropique tandis qu'il est ici un gaz parfait (où donc l'énergie interne, ou la température, est une inconnue supplémentaire). Nous identifions le système limite et proposons un algorithme pour le résoudre de manière approchée.

La suite de ce travail, en cours, concerne l'écriture d'un algorithme de résolution du système non limite, et l'étude des propriétés asymptotiques dudit schéma.
\end{abstract}

\section{INTRODUCTION}

We are interested in a PDE system describing the interaction between a fluid and a set of droplets immersed in the fluid. This situation occurs in combustion theory [15], motivated for instance by the design of engines or propulsors [11]. We also mention the dynamics of sprays with many applications e.g. biomedical sprays [2], dispersion of pollutants [13], the optimization of fine water spray fire suppression systems... The fluid is described by the evolution of its density $\rho(t, x) \geq 0$, its velocity $u(t, x) \in \mathbb{R}^{N}(N=1,2$ or 3$)$ and its total energy

\footnotetext{
1 UPMC Paris 06, Lab. J.-L. Lions, 175 rue du Chevaleret, BC 187, F-75013 Paris, France; e-mail: laurent.boudin@upmc.fr \& boutin@ann.jussieu.fr

2 INRIA Paris-Rocquencourt, REO Project team, BP 105, F-78153 Le Chesnay Cedex, France.

3 CEA, DEN/DANS/DM2S/SFME/LETR, F-91191 Gif-sur-Yvette, France.

4 DTIM/ONERA Centre de Toulouse, 2 avenue Edouard Belin, 31055 Toulouse, France. e-mail: bruno.fornet@onera.fr

5 Project-Team SIMPAF, INRIA Lille Nord Europe Research Centre Park Plazza, 40 avenue Halley 59650 Villeneuve d'Ascq CEDEX, France; e-mail: thierry.goudon@inria.fr \& pauline.lafitte-godillon@math.univ-lille1.fr

\& lagoutie@math.jussieu.fr

6 Laboratoire Paul Painlevé, USTL-CNRS UMR 8524, Cité Scientifique, 59655 Villeneuve d'Ascq CEDEX, France.

7 Université Paris Diderot-Paris 7, Lab. J.-L. Lions, 175 rue du Chevaleret, BC 187, F-75013 Paris, France.

8 Université Paris Nord - Institut Galilée LAGA (Laboratoire d'Analyse, Géométrie et Applications) Avenue J.B. Clément 93430 Villetaneuse, France; e-mail: merlet@math.univ-paris13.fr

(C) EDP Sciences, SMAI 2009
} 
$E(t, x) \geq 0$, which are functions of time $t \geq 0$ and position $x \in \mathbb{R}^{N}$. We define the internal energy $e$, the pressure $p$, the temperature $\Theta$ by the relations

$$
e=\frac{p}{(\gamma-1) \rho} \geq 0, \quad p=R \rho \Theta, \quad E=e+\frac{u^{2}}{2}
$$

where $R$ is the perfect gas constant and $\gamma>1$ is the adiabatic constant. The disperse phase is described by its density distribution in phase space $f(t, x, v) \geq 0$, where the variable $v \in \mathbb{R}^{N}$ stands for the velocity of the particles. Macroscopic quantities can be defined as moments with respect to $v$; in what follows we need

$$
\begin{cases}\text { the macroscopic density: } & n(t, x)=\int_{\mathbb{R}^{N}} f(t, x, v) \mathrm{d} v, \\ \text { the bulk velocity: } & n V(t, x)=\int_{\mathbb{R}^{N}} v f(t, x, v) \mathrm{d} v, \\ \text { the temperature: } & n|V|^{2}(t, x)+N n \Theta_{p}=\int_{\mathbb{R}^{N}}|v|^{2} f(t, x, v) \mathrm{d} v, \\ \text { the heat flux: } & q(t, x)=\int_{\mathbb{R}^{N}} v \frac{|v|^{2}}{2} f(t, x, v) \mathrm{d} v .\end{cases}
$$

The evolution of the density $f$ is governed by

$$
\partial_{t} f+v \cdot \nabla_{x} f=\operatorname{div}_{v}\left((v-u) f+\Theta \nabla_{v} f\right)-\eta_{p} \nabla_{x} \Phi \cdot \nabla_{v} f
$$

The divergence term in the right-hand side accounts for both the friction force exerted by the fluid on the particles, which is supposed to be proportional to the relative velocity $(v-u)$, and the Brownian motion of the particles, which induces diffusion with respect to the velocity variable, depending on the surrounding temperature $\Theta$. Note that more complicated and nonlinear expressions of the drag force can be used; the linear relation with respect to the relative velocity applies when the Reynolds number of the flow is low. The second term in the right-hand side comes from an external force with potential $\Phi$ and $\eta_{p}$ is a positive constant. The evolution of the fluid obeys the Euler system

$$
\left\{\begin{array}{l}
\partial_{t} \rho+\operatorname{div}_{x}(\rho u)=0 \\
\partial_{t}(\rho u)+\operatorname{Div}_{x}(\rho u \otimes u)+\nabla_{x} p=\mathscr{F}-\eta_{f} \rho \nabla_{x} \Phi \\
\partial_{t}(\rho E)+\operatorname{div}_{x}((\rho E+p) u)=\mathscr{E}-\eta_{f} \rho u \cdot \nabla_{x} \Phi
\end{array}\right.
$$

where $\eta_{f}$ is another positive coefficient that accounts for a possible difference of amplitude in the forces applied to the fluid or the disperse phase. Remark that assuming $\eta_{p}$ and $\eta_{f}$ positive means that the external force associated to the potential $\Phi$ acts on opposite directions on the particles and on the fluid. Bearing in mind the example of gravity we have $\nabla_{x} \Phi=g \in \mathbb{R}^{N}$ and we are dealing with a situation where particles are light compared to the fluid: gravity pushes the fluid downward, while buoyancy effects push the particles upward. In other words, here and below, the disperse phase is buoyancy driven, while the fluid is gravity driven. In such a situation, we can expect the formation of sedimentation profiles. We refer to [3], [4] for a discussion on this modeling issue. Here, the main point we address is the introduction of the energy equation and the description of energy exchanges.

In $(2)$, the force $\mathscr{F}$ arising in the right-hand side of the momentum equation is given by the friction force exerted by the particles on the fluid and it reads

$$
\mathscr{F}(t, x)=n(V-u)(t, x)=\int_{\mathbb{R}^{N}}(v-u) f(t, x, v) \mathrm{d} v
$$


The energy exchanges between the two phases split as follows:

$$
\begin{aligned}
\mathscr{E}(t, x)= & \left(n(V-u) \cdot u+N n\left(\Theta_{p}-\Theta\right)\right)(t, x)+\mathscr{E}^{\prime}(t, x) \\
= & \int_{\mathbb{R}^{N}}(v-u) f(t, x, v) \mathrm{d} v \cdot u \\
& +N\left(\Theta_{p}-\Theta\right)(t, x) \int_{\mathbb{R}^{N}} f(t, x, v) \mathrm{d} v+\mathscr{E}^{\prime}(t, x) .
\end{aligned}
$$

In this expression, the first term is nothing but the work of the friction force $\mathscr{F}$ while the second describes the heat transfer between the two phases; the last term, which will be specified later on, guarantees the total energy conservation. As a matter of fact, we remark that the evolution of the internal energy is driven by

$$
\partial_{t}(\rho e)+\operatorname{div}_{x}(\rho e u)+p \operatorname{div}_{x} u=N n\left(\Theta_{p}-\Theta\right)+\mathscr{E}^{\prime}(t, x) .
$$

In this modeling, we disregard many physical effects that could be non negligible for applications (added mass effects, Basset forces...). Nevertheless in most cases, these effects are of many orders smaller than the drag and gravity forces. We restrict ourselves to the description of thin sprays which means that

- we neglect inter-particles collisions and coagulation-fragmentation phenomena.

- the volume fraction of the particles is neglected as well.

We refer for instance to [12] for further discussions of the modeling issues.

In this paper, the goal is to extend, at least formally, the analysis performed in [3] (see also [9, 10]) and, having identified relevant asymptotic regimes, to design adapted Asymptotic Preserving schemes, in the spirit of $[4,5,8]$. The dissipative or relaxation properties of the system are crucial to this approach.

\section{Dissipation properties}

Let us start by considering the evolution of the macroscopic quantities

$$
\begin{aligned}
& \partial_{t} n+\operatorname{div}_{x}(n V)=0 \\
& \partial_{t}(n V)+\operatorname{Div}_{x}\left(\int_{\mathbb{R}^{N}} v \otimes v f \mathrm{~d} v\right)=-n(V-u)+\eta_{p} n \nabla_{x} \Phi, \\
& \partial_{t}\left(\int_{\mathbb{R}^{N}} \frac{v^{2}}{2} f \mathrm{~d} v\right)+\operatorname{div}_{x} q=-\int_{\mathbb{R}^{N}}(v-u) \cdot v f \mathrm{~d} v+N n \Theta+\eta_{p} n V \cdot \nabla_{x} \Phi,
\end{aligned}
$$

where we use integration by parts for evaluating the right-hand sides. Observe that the total momentum is conserved (up to the gravity term) since

$$
\partial_{t}(\rho u+n V)+\operatorname{Div}_{x}\left(\rho u \otimes u+\int_{\mathbb{R}^{N}} v \otimes v f \mathrm{~d} v\right)+\nabla_{x} p=\left(\eta_{p} n-\eta_{f} \rho\right) \nabla_{x} \Phi .
$$

Next, since we consider the mixture fluid/particles as a whole, the total energy should also be conserved, which will give the definition of $\mathscr{E} '$. We remark that

$$
\begin{aligned}
\int_{\mathbb{R}^{N}}(v-u) \cdot v f \mathrm{~d} v & =\int_{\mathbb{R}^{N}}|v-u|^{2} f \mathrm{~d} v+n(V-u) \cdot u \\
& =n\left(|V|^{2}+N \Theta_{p}-2 V \cdot u+|u|^{2}\right)+n(V-u) \cdot u \\
& =n|V-u|^{2}+N n \Theta_{p}+n(V-u) \cdot u
\end{aligned}
$$

Therefore the kinetic energy of the particles obeys

$$
\partial_{t}\left(\int_{\mathbb{R}^{N}} \frac{|v|^{2}}{2} f \mathrm{~d} v\right)+\operatorname{div}_{x} q=-n|V-u|^{2}+N n\left(\Theta-\Theta_{p}\right)-n(V-u) \cdot u+\eta_{p} n V \cdot \nabla_{x} \Phi .
$$


Accordingly, we set

$$
\mathscr{E}^{\prime}(t, x)=n|V-u|^{2}
$$

so that the total energy is driven by

$$
\partial_{t}\left(\rho E+\int_{\mathbb{R}^{N}} \frac{|v|^{2}}{2} f \mathrm{~d} v\right)+\operatorname{div}_{x}((\rho E+p) u+q)=\left(\eta_{p} n V-\eta_{f} \rho u\right) \cdot \nabla_{x} \Phi
$$

Hence, the term $\mathscr{E}^{\prime}$ appears as a source of internal energy, or a source of heat, for the fluid, produced by the friction with the particle, and proportional to the macroscopic kinetic energy defined by the relative velocity $V-u$.

Next, we consider the entropy $S(t, x)$ defined by the relation

$$
S=-\frac{R}{\gamma-1} \ln \left(p \rho^{-\gamma}\right)=-\frac{R}{\gamma-1} \ln \left(R \frac{\Theta}{\rho^{\gamma-1}}\right) .
$$

We check that it satisfies

$$
\begin{aligned}
\partial_{t}(\rho S)+\operatorname{div}_{x}(\rho S u) & =\frac{R \rho}{p}\left(n(V-u) \cdot u-n(V-u) \cdot u-N n\left(\Theta_{p}-\Theta\right)-\mathscr{E}^{\prime}\right) \\
& =-N n\left(\frac{\Theta_{p}}{\Theta}-1\right)-\frac{n}{\Theta}|V-u|^{2}
\end{aligned}
$$

Finally, we look at the entropy of the disperse phase

$$
\begin{aligned}
\frac{\mathrm{d}}{\mathrm{d} t} \int_{\mathbb{R}^{N}} \int_{\mathbb{R}^{N}} f \ln (f) \mathrm{d} v \mathrm{~d} x & =-\int_{\mathbb{R}^{N}} \int_{\mathbb{R}^{N}}\left((v-u) f \cdot \frac{\nabla_{v} f}{f}+\Theta \frac{\left|\nabla_{v} f\right|^{2}}{f}\right) \mathrm{d} v \mathrm{~d} x \\
& =N \int_{\mathbb{R}^{N}} n \mathrm{~d} x-\int_{\mathbb{R}^{N}} \int_{\mathbb{R}^{N}} \Theta \frac{\left|\nabla_{v} f\right|^{2}}{f} \mathrm{~d} v \mathrm{~d} x
\end{aligned}
$$

Hence, the total entropy satisfies

$$
\begin{array}{r}
\frac{\mathrm{d}}{\mathrm{d} t}\left(\int_{\mathbb{R}^{N}} \rho S \mathrm{~d} x+\int_{\mathbb{R}^{N}} \int_{\mathbb{R}^{N}} f \ln (f) \mathrm{d} v \mathrm{~d} x\right) \\
=-\int_{\mathbb{R}^{N}} \int_{\mathbb{R}^{N}} \Theta \frac{\left|\nabla_{v} f\right|^{2}}{f} \mathrm{~d} v \mathrm{~d} x+2 N \int_{\mathbb{R}^{N}} n \mathrm{~d} x \\
-N \int_{\mathbb{R}^{N}} n \frac{\Theta_{p}}{\Theta} \mathrm{d} x-\int_{\mathbb{R}^{N}} \frac{n|V-u|^{2}}{\Theta} \mathrm{d} x
\end{array}
$$

The next argument is two-fold. On the one hand, we observe that

$$
N \int_{\mathbb{R}^{N}} n \mathrm{~d} x=-\int_{\mathbb{R}^{N}} \int_{\mathbb{R}^{N}} \frac{v-V}{\sqrt{\Theta}} \sqrt{f} \cdot \sqrt{\Theta} \frac{\nabla_{v} f}{\sqrt{f}} \mathrm{~d} v \mathrm{~d} x
$$

and on the other hand we have

$$
\int_{\mathbb{R}^{N}}|v-V|^{2} f \mathrm{~d} v=n|V|^{2}+N n \Theta_{p}-2 n V \cdot V+n|V|^{2}=N n \Theta_{p}
$$


It follows that

$$
\begin{aligned}
\frac{\mathrm{d}}{\mathrm{d} t}\left(\int_{\mathbb{R}^{N}} \rho S \mathrm{~d} x+\int_{\mathbb{R}^{N}} \int_{\mathbb{R}^{N}} f \ln (f) \mathrm{d} v \mathrm{~d} x\right) \\
=-\int_{\mathbb{R}^{N}} \int_{\mathbb{R}^{N}}\left(\Theta \frac{\left|\nabla_{v}\right|^{2}}{f}+2 \frac{v-V}{\sqrt{\Theta}} \sqrt{f} \cdot \sqrt{\Theta} \frac{\nabla_{v} f}{\sqrt{f}}+\frac{|v-V|^{2}}{\Theta} f\right) \mathrm{d} v \mathrm{~d} x \\
\quad-\int_{\mathbb{R}^{N}} n \frac{|V-u|^{2}}{\Theta} \mathrm{d} x \\
=-\int_{\mathbb{R}^{N}} \int_{\mathbb{R}^{N}}\left|\sqrt{\Theta} \frac{\nabla_{v} f}{\sqrt{f}}+\frac{v-V}{\sqrt{\Theta}} \sqrt{f}\right|^{2} \mathrm{~d} v \mathrm{~d} x-\int_{\mathbb{R}^{N}} n \frac{|V-u|^{2}}{\Theta} \mathrm{d} x,
\end{aligned}
$$

which indicates that the total entropy of the system is dissipated.

Let us summarize the computation as follows.

Proposition 1. Let $(\rho, u, E, f)$ be a (smooth enough) solution of (1)-(4). Then, both the total momentum and the total energy are conserved while the total entropy is dissipated and we have

$$
\begin{aligned}
& \frac{\mathrm{d}}{\mathrm{d} t}\left(\int_{\mathbb{R}^{N}} \rho E \mathrm{~d} x+\int_{\mathbb{R}^{N}} \int_{\mathbb{R}^{N}} \frac{v^{2}}{2} f \mathrm{~d} v \mathrm{~d} x\right)=\int_{\mathbb{R}^{N}}\left(\eta_{p} n V-\eta_{f} \rho u\right) \cdot \nabla_{x} \Phi \mathrm{d} x, \\
& \frac{\mathrm{d}}{\mathrm{d} t}\left(\int_{\mathbb{R}^{N}} \rho S \mathrm{~d} x+\int_{\mathbb{R}^{N}} \int_{\mathbb{R}^{N}} f \ln (f) \mathrm{d} v \mathrm{~d} x\right) \\
& =-\int_{\mathbb{R}^{N}} \int_{\mathbb{R}^{N}}\left|\sqrt{\Theta} \frac{\nabla_{v}^{f}}{\sqrt{f}}+\frac{v-V}{\sqrt{\Theta}} \sqrt{f}\right|^{2} \mathrm{~d} v \mathrm{~d} x-\int_{\mathbb{R}^{N}} \int_{\mathbb{R}^{N}} f \frac{|V-u|^{2}}{\Theta} \mathrm{d} v \mathrm{~d} x \leq 0 .
\end{aligned}
$$

Note that the dissipation terms vanish when

$$
u=V \quad \text { and } \quad f(t, x, v)=\frac{n(t, x)}{(2 \pi \Theta(t, x))^{N / 2}} \exp \left(-\frac{|v-V(t, x)|^{2}}{2 \Theta(t, x)}\right) .
$$

\section{Bubbling Regime}

According to [3], the "Bubbling Regime" relies on the following scaling:

$$
\left\{\begin{array}{l}
\partial_{t} f^{\epsilon}+\frac{1}{\epsilon} v \cdot \nabla_{x} f^{\epsilon}=\frac{1}{\epsilon^{2}} \operatorname{div}_{v}\left(\left(v-\epsilon u^{\epsilon}\right) f^{\epsilon}+\Theta^{\epsilon} \nabla_{v} f^{\epsilon}\right)-\frac{1}{\epsilon} \nabla_{x} \Phi \cdot \nabla_{v} f^{\epsilon}, \\
\partial_{t} \rho^{\epsilon}+\operatorname{div}_{x}\left(\rho^{\epsilon} u^{\epsilon}\right)=0 \\
\partial_{t}\left(\rho^{\epsilon} u^{\epsilon}\right)+\operatorname{Div}_{x}\left(\rho^{\epsilon} u^{\epsilon} \otimes u^{\epsilon}\right)+\nabla_{x} p^{\epsilon}=\frac{1}{\epsilon} \int_{\mathbb{R}^{N}}\left(v-\epsilon u^{\epsilon}\right) f^{\epsilon} \mathrm{d} v-\frac{1}{1-\epsilon^{2}} \rho^{\epsilon} \nabla_{x} \Phi \\
\partial_{t}\left(\rho^{\epsilon} E^{\epsilon}\right)+\operatorname{div}_{x}\left(\left(\rho^{\epsilon} E^{\epsilon}+p^{\epsilon}\right) u^{\epsilon}\right) \\
\quad=\frac{1}{\epsilon^{2}}\left(\int_{\mathbb{R}^{N}}\left(v-\epsilon u^{\epsilon}\right) f^{\epsilon} \mathrm{d} v \cdot \epsilon u^{\epsilon}+N n^{\epsilon}\left(\Theta_{p}^{\epsilon}-\Theta^{\epsilon}\right)+n^{\epsilon}\left|V^{\epsilon}-\epsilon u^{\epsilon}\right|^{2}\right)-\frac{1}{1-\epsilon^{2}} \rho^{\epsilon} u^{\epsilon} \cdot \nabla_{x} \Phi
\end{array}\right.
$$

where it is convenient to introduce the following notation

$$
\begin{array}{llrl}
n^{\epsilon}=\int_{\mathbb{R}^{N}} f^{\epsilon} \mathrm{d} v, & J^{\epsilon}=\frac{1}{\epsilon} n^{\epsilon} V^{\epsilon}=\int_{\mathbb{R}^{N}} \frac{v}{\epsilon} f^{\epsilon} \mathrm{d} v, \\
n^{\epsilon}\left|V^{\epsilon}\right|^{2}+N n^{\epsilon} \Theta_{p}^{\epsilon}=\int_{\mathbb{R}^{N}} v^{2} f^{\epsilon} \mathrm{d} v, & q^{\epsilon}=\int_{\mathbb{R}^{N}} \frac{v}{\epsilon} \frac{v^{2}}{2} f^{\epsilon} \mathrm{d} v .
\end{array}
$$

The scaling can be explained as follows. Given the radius $d$ of the particles, the particles density $\rho_{p}$ and the typical fluid density $\rho_{f}$, the dynamic viscosity of the fluid $\mu$ we define the Stokes settling time

$$
\tau_{S}=\frac{2 \rho_{p} d^{2}}{9 \mu}
$$


and the Stokes settling velocity

$$
V_{S}=\tau_{S} g\left|1-\rho_{f} / \rho_{p}\right| .
$$

Dropping a particle in the fluid at rest, $V_{S}$ is the asymptotic velocity of the particle, and $\tau_{S}$ the corresponding relaxation time. Hence, $\tau_{S}$ characterizes the strength of the drag force and $V_{S}$ characterizes the effect of the external force, here gravity-buoyancy, on the particles. Then, we introduce time and length scales of observation, $T$ and $L$ respectively and we set $U=L / T$ which stands for the velocity unit. It is the typical velocity of the fluid. It has to be compared to both $V_{S}$ and the thermal velocity

$$
V_{t h}=\sqrt{\frac{3 k \Theta}{4 \pi \rho_{p} d^{3}}},
$$

with $\Theta$ a typical value of the temperature and $k$ the Boltzmann constant. The scaling assumes the following relations

$$
\tau_{S}=\epsilon^{2} T \ll T, \quad V_{S} \simeq U=\epsilon V_{t h}, \quad \rho_{p}=\epsilon \rho_{f} .
$$

Therefore, the Richardson number, which is the ratio of potential to kinetic energy, is $1 /\left(1-\epsilon^{2}\right)$ for the fluid and $1 / \epsilon$ for the particles. Similarly, the temperature relaxation is characterized by

$$
\tau_{t h}=\frac{1}{N u 4 \pi d \Lambda \Theta}
$$

where $N u$ is the Nusselt number, and $\Lambda$ the thermal conductivity. We remind that the Nusselt number is a dimensionless ratio of convection heat transfer to fluid conduction heat transfer. In the present context, we have the following relation

$$
\tau_{t h}=\epsilon^{2} T .
$$

Of course, many other different scaling are relevant, as described in the above mentioned references. Nevertheless, we restrict ourselves to this regime, extension of the bubbling regime considered in $[3,4]$. Our goal is two-fold:

- firstly, we derive the asymptotic hydrodynamic system corresponding to the limit $\epsilon \rightarrow 0$,

- secondly, we design a numerical scheme for the limit system and comment on numerics the influence of the energy exchanges.

In this context Proposition 1 recasts as

$$
\begin{aligned}
& \frac{\mathrm{d}}{\mathrm{d} t}\left(\int_{\mathbb{R}^{N}} \rho^{\epsilon} E^{\epsilon} \mathrm{d} x+\int_{\mathbb{R}^{N}} \int_{\mathbb{R}^{N}} \frac{v^{2}}{2} f^{\epsilon} \mathrm{d} v \mathrm{~d} x\right)=\int_{\mathbb{R}^{N}}\left(\frac{n^{\epsilon} V^{\epsilon}}{\epsilon}-\frac{\rho^{\epsilon} u^{\epsilon}}{1-\epsilon^{2}}\right) \cdot \nabla_{x} \Phi \mathrm{d} x, \\
& \frac{\mathrm{d}}{\mathrm{d} t}\left(\int_{\mathbb{R}^{N}} \rho^{\epsilon} S^{\epsilon} \mathrm{d} x+\int_{\mathbb{R}^{N}} \int_{\mathbb{R}^{N}} f^{\epsilon} \ln \left(f^{\epsilon}\right) \mathrm{d} v \mathrm{~d} x\right) \\
& \quad+\frac{1}{\epsilon^{2}} \int_{\mathbb{R}^{N}} \int_{\mathbb{R}^{N}}\left[\left|\sqrt{\Theta^{\epsilon}} \frac{\nabla_{v} f^{\epsilon}}{\sqrt{f^{\epsilon}}}+\frac{v-V^{\epsilon}}{\sqrt{\Theta^{\epsilon}}} \sqrt{f^{\epsilon}}\right|^{2}+f^{\epsilon}\left|V^{\epsilon}-\epsilon u^{\epsilon}\right|^{2}\right] \mathrm{d} v \mathrm{~d} x \leq 0,
\end{aligned}
$$

and we infer the following relaxation effects

$$
\begin{aligned}
& V^{\epsilon} \simeq \epsilon u^{\epsilon} \\
& f^{\epsilon}(t, x, v) \simeq \frac{n^{\epsilon}(t, x)}{\left(2 \pi \Theta^{\epsilon}(t, x)\right)^{N / 2}} \exp \left(-\frac{\left|v-V^{\epsilon}(t, x)\right|^{2}}{2 \Theta^{\epsilon}(t, x)}\right) \simeq n^{\epsilon}(t, x) M_{\Theta^{\epsilon}(t, x)}(v)
\end{aligned}
$$

where $M_{\Theta}$ stands for the centered Maxwellian with temperature $\Theta$

$$
M_{\Theta}(v)=(2 \pi \Theta)^{-N / 2} e^{-v^{2} /(2 \Theta)} .
$$


Accordingly, we obtain

$$
\int_{\mathbb{R}^{N}} v^{2} f^{\epsilon} \mathrm{d} v=n^{\epsilon}\left|V^{\epsilon}\right|^{2}+N n^{\epsilon} \Theta_{p}^{\epsilon} \simeq \int_{\mathbb{R}^{N}} v^{2} n^{\epsilon} M_{\Theta^{\epsilon}} \mathrm{d} v=N n^{\epsilon} \Theta^{\epsilon}
$$

with $V^{\epsilon}$ expected to be of order $\mathcal{O}(\epsilon)$. We deduce that

$$
\Theta^{\epsilon}-\Theta_{p}^{\epsilon} \underset{\epsilon \rightarrow 0}{\longrightarrow} 0
$$

(an observation which can be seen also by considering the energy equation). Eventually, we guess that the asymptotic behavior is described by the evolution of the macroscopic quantities $n, \rho, u, \Theta$ only, and we assume that

$$
\left(n^{\epsilon}, \rho^{\epsilon}, u^{\epsilon}, \Theta^{\epsilon}\right) \underset{\epsilon \rightarrow 0}{\longrightarrow}(n, \rho, u, \Theta)
$$

(in a strong enough sense...), and consequently

$$
f^{\epsilon}(t, x, v) \rightarrow n(t, x) M_{\Theta(t, x)}(v)
$$

It thus remains to determine the equations satisfied by these quantities.

To this end, we go back to the moments equations (5) which recast here in the following rescaled form

$$
\left\{\begin{aligned}
& \partial_{t} n^{\epsilon}+\operatorname{div}_{x}\left(J^{\epsilon}\right)= 0 \\
& \epsilon^{2} \partial_{t} J^{\epsilon}+\operatorname{Div}_{x}\left(\int_{\mathbb{R}^{N}} v \otimes v f^{\epsilon} \mathrm{d} v\right)=-J^{\epsilon}+n^{\epsilon} u^{\epsilon}+n^{\epsilon} \nabla_{x} \Phi \\
& \partial_{t}\left(\int_{\mathbb{R}^{N}} \frac{v^{2}}{2} f^{\epsilon} \mathrm{d} v\right)+\operatorname{div}_{x} q^{\epsilon}=\frac{1}{\epsilon^{2}}\left(-\int_{\mathbb{R}^{N}}\left(v-\epsilon u^{\epsilon}\right) \cdot v f^{\epsilon} \mathrm{d} v+N n^{\epsilon} \Theta^{\epsilon}\right)-\frac{1}{\epsilon} \nabla_{x} \cdot \int_{\mathbb{R}^{N}} \frac{|v|^{2}}{2} \nabla_{v} f^{\epsilon} \mathrm{d} v \\
&=\frac{1}{\epsilon^{2}} n^{\epsilon}\left(-\left|V^{\epsilon}\right|^{2}-N \Theta_{p}^{\epsilon}+\epsilon u^{\epsilon} \cdot V^{\epsilon}+N \Theta^{\epsilon}\right)+\frac{n^{\epsilon} V^{\epsilon}}{\epsilon} \cdot \nabla_{x} \Phi \\
&=N n^{\epsilon} \frac{\Theta^{\epsilon}-\Theta_{p}^{\epsilon}}{\epsilon^{2}}+J^{\epsilon} \cdot\left(u^{\epsilon}-\frac{J^{\epsilon}}{n^{\epsilon}}+\nabla_{x} \Phi\right)
\end{aligned}\right.
$$

We also suppose that

$$
\left(J^{\epsilon}, q^{\epsilon}\right) \underset{\epsilon \rightarrow 0}{\longrightarrow}(J, q)
$$

holds and we wish to relate these limits to $(n, \rho, u, \Theta)$. The ansatz (12) allows to compute the limit of the second moment

$$
\int_{\mathbb{R}^{N}} v \otimes v f^{\epsilon} \mathrm{d} v \underset{\epsilon \rightarrow 0}{\longrightarrow} n \int_{\mathbb{R}^{N}} v \otimes v M_{\Theta}(v) \mathrm{d} v=n \Theta \mathbb{I} .
$$

Therefore, letting $\epsilon$ go to 0 in the momentum equation leads to

$$
0+\nabla_{x}(n \Theta)=-J+n\left(u+\nabla_{x} \Phi\right)
$$

Next, we consider the equation for the total energy

$$
\partial_{t}\left(\rho^{\epsilon} E^{\epsilon}+\int_{\mathbb{R}^{N}} \frac{|v|^{2}}{2} f^{\epsilon} \mathrm{d} v\right)+\operatorname{div}_{x}\left(\left(\rho^{\epsilon} E^{\epsilon}+p^{\epsilon}\right) u^{\epsilon}+q^{\epsilon}\right)=\left(J^{\epsilon}-\frac{\rho^{\epsilon} u^{\epsilon}}{1-\epsilon^{2}}\right) \cdot \nabla_{x} \Phi .
$$

As $\epsilon \rightarrow 0$ we get

$$
\partial_{t}\left(\rho E+\frac{N}{2} n \Theta\right)+\operatorname{div}_{x}((\rho E+p) u+q)=(J-\rho u) \cdot \nabla_{x} \Phi .
$$

We are left with the task of identifying the heat flux $q$. To this purpose, we observe that

$$
\epsilon^{2} \partial_{t} q^{\epsilon}+\operatorname{Div}_{x}\left(\int_{\mathbb{R}^{N}} v \otimes v \frac{|v|^{2}}{2} f^{\epsilon} \mathrm{d} v\right)=-3 q^{\epsilon}+\left(\int_{\mathbb{R}^{N}}\left(v \otimes v+\frac{|v|^{2}}{2} \mathbb{I}\right) f^{\epsilon} \mathrm{d} v\right) u^{\epsilon}+(N+2) \Theta^{\epsilon} J^{\epsilon}-\int_{\mathbb{R}^{N}} v \frac{|v|^{2}}{2}\left(\nabla_{v} f \cdot \nabla_{x} \Phi\right) \mathrm{d} v
$$


Hence, when $\epsilon$ goes to 0 we find

$$
\begin{aligned}
0+\nabla_{x}( & \left.\frac{n}{2 N} \int_{\mathbb{R}^{N}}|v|^{4} M_{\Theta}(v) \mathrm{d} v\right)=\nabla_{x}\left(\frac{N+2}{2} n \Theta^{2}\right) \\
& =\frac{N^{2} 2}{2} \Theta^{2} \nabla_{x} n+(N+2) n \Theta \nabla_{x} \Theta \\
& =-3 q+\frac{N+2}{2} n \Theta\left(u+\nabla_{x} \Phi\right)+(N+2) \Theta\left(n\left(u+\nabla_{x} \Phi\right)-\nabla_{x}(n \Theta)\right) \\
& =-3 q+\frac{3}{2}(N+2) n \Theta\left(u+\nabla_{x} \Phi\right)-(N+2)\left(n \Theta \nabla_{x} \Theta+\Theta^{2} \nabla_{x} n\right) .
\end{aligned}
$$

since $\int_{\mathbb{R}^{N}}|v|^{4} M_{1}(v) \mathrm{d} v=N(N+2)$. It defines the heat flux $q$ by means of $n, u$ and $\Theta$ :

$$
q=\frac{N+2}{2} n \Theta\left(u+\nabla_{x} \Phi\right)-\frac{N+2}{2} \Theta^{2} \nabla_{x} n-\frac{2(N+2)}{3} n \Theta \nabla_{x} \Theta .
$$

The conclusion of the computations states as follows.

Theorem 1. Assuming strong enough convergence of the macroscopic quantities, the limit as $\epsilon$ goes to 0 is described by the following set of PDEs

$$
\left\{\begin{array}{l}
\partial_{t} \rho+\operatorname{div}_{x}(\rho u)=0 \\
\partial_{t} n+\operatorname{div}_{x}\left(n u-\nabla_{x}(n \Theta)\right)=-\operatorname{div}_{x}\left(n \nabla_{x} \Phi\right) \\
\partial_{t}(\rho u)+\operatorname{Div}_{x}(\rho u \otimes u+p)=-\nabla_{x}(n \Theta)+(n-\rho) \nabla_{x} \Phi \\
\partial_{t}\left(\rho E+\frac{N}{2} n \Theta\right)+\operatorname{div}_{x}\left((\rho E+p) u+\frac{N+2}{2} n \Theta u\right) \\
\quad=\frac{N+2}{2} \operatorname{div}_{x}\left(\Theta^{2} \nabla_{x} n\right)+\frac{2(N+2)}{3} \operatorname{div}_{x}\left(n \Theta \nabla_{x} \Theta\right) \\
\quad-\frac{N+2}{2} \operatorname{div}_{x}\left(n \Theta \nabla_{x} \Phi\right)+(n-\rho) u \nabla_{x} \Phi+n\left|\nabla_{x} \Phi\right|^{2}-\nabla_{x} \Phi \cdot \nabla_{x}(n \Theta) .
\end{array}\right.
$$

In System (17) the unknowns are the fluid density $\rho$, the particle (macroscopic) density $n$ and the common velocity $u$ and temperature $\Theta$. Assuming a constant temperature and dropping the energy equation, we recover the equations derived in [3]. There appear some unusual terms in the equations which are reminiscent of the so-called Soret and Dufour effects, see [14]. The Soret effect relies on the conduction current $n \nabla_{x} \Theta$ : the temperature gradient produces a flow of particles. Note that particles are also subject to diffusion with a diffusion coefficient proportional to the temperature. The Dufour effect relies on the effects of concentration gradients on the evolution of the temperature.

Another way of deriving the limit equations consists in considering fluctuations in the ansatz (12). This approach motivates the design of "Asymptotic Preserving" schemes, see $[4,5,8]$. Namely, we set

$$
f^{\epsilon}(t, x, v)=n^{\epsilon}(t, x) M_{\Theta^{\epsilon}(t, x)}(v)+\epsilon r^{\epsilon}(t, x, v)
$$

and we wish to identify the limit $r$ of $\left(r^{\epsilon}\right)$ by using the equation

$$
\epsilon \partial_{t} f^{\epsilon}+v \cdot \nabla_{x} f^{\epsilon}=\mathscr{L}_{\Theta^{\epsilon}} r^{\epsilon}-u^{\epsilon} \cdot \nabla_{v} f^{\epsilon}-\nabla_{x} \Phi \cdot \nabla_{v} f^{\epsilon}
$$

where $\mathscr{L}_{\Theta}$ stands for the Fokker-Planck operator

$$
\mathscr{L}_{\Theta} f=\operatorname{div}_{v}\left(v f+\Theta \nabla_{v} f\right)
$$

(recall that $\mathscr{L}_{\Theta}\left(M_{\Theta}\right)=0$ for every $\Theta \in \mathbb{R}$ ). Indeed, assuming that $r^{\epsilon}$ converges to $r$, we can express the mass and heat fluxes as follows

$$
J^{\epsilon}=\int_{\mathbb{R}^{N}} v r^{\epsilon} \mathrm{d} v \underset{\epsilon \rightarrow 0}{\longrightarrow} J=\int_{\mathbb{R}^{N}} v r \mathrm{~d} v, \quad q^{\epsilon}=\int_{\mathbb{R}^{N}} v \frac{|v|^{2}}{2} r^{\epsilon} \mathrm{d} v \underset{\epsilon \rightarrow 0}{\longrightarrow} q=\int_{\mathbb{R}^{N}} v \frac{|v|^{2}}{2} r \mathrm{~d} v .
$$


In the the last two terms of (18), the leading contribution reads

$$
-\left(u^{\epsilon}+\nabla_{x} \Phi\right) \cdot \nabla_{v}\left(n^{\epsilon} M_{\Theta^{\epsilon}}\right)=+\left(u^{\epsilon}+\nabla_{x} \Phi\right) \cdot \frac{v}{\Theta^{\epsilon}} n^{\epsilon} M_{\Theta^{\epsilon}} .
$$

Therefore, $r$ is characterized by the relation

$$
\begin{aligned}
\mathscr{L}_{\Theta} r & =v \cdot \nabla_{x}\left(n M_{\Theta}\right)-\left(u+\nabla_{x} \Phi\right) \cdot \frac{v}{\Theta} n M_{\Theta} \\
& =v M_{\Theta} \cdot\left(\nabla_{x} n-\left(u+\nabla_{x} \Phi\right) \frac{n}{\Theta}-\frac{N}{2} \frac{n}{\Theta} \nabla_{x} \Theta\right)+v \frac{|v|^{2}}{2} M_{\Theta} \cdot \frac{n}{\Theta^{2}} \nabla_{x} \Theta .
\end{aligned}
$$

The solution splits as follows

$$
\begin{aligned}
& r=r^{1}+r^{2} \\
& r^{1}(t, x, v)=\chi(t, x, v) \cdot\left(\nabla_{x} n-\left(u+\nabla_{x} \Phi\right) \frac{n}{\Theta}-\frac{N}{2} \frac{n}{\Theta} \nabla_{x} \Theta\right) \\
& r^{2}(t, x, v)=\lambda(t, x, v) \cdot \frac{n}{\Theta^{2}} \nabla_{x} \Theta
\end{aligned}
$$

where $\chi=\left(\chi_{1}, \ldots, \chi_{N}\right)$ and $\lambda=\left(\lambda_{1}, \ldots, \lambda_{N}\right)$ are solutions of the auxiliary problems (where $t, x$ appear only as parameters through the temperature)

$$
\mathscr{L}_{\Theta(t, x)} \chi_{j}=v_{j} M_{\Theta(t, x)}, \quad \mathscr{L}_{\Theta(t, x)} \lambda_{j}=v_{j} \frac{|v|^{2}}{2} M_{\Theta(t, x)}
$$

We check readily that

$$
\chi=-v M_{\Theta}, \quad \lambda=-v M_{\Theta} \frac{1}{3}\left(\frac{|v|^{2}}{2}+(N+2) \Theta\right) .
$$

The associated mass fluxes are given by

$$
\begin{aligned}
J_{\chi} & =\int_{\mathbb{R}^{N}} v \otimes \chi \mathrm{d} v=-\int_{\mathbb{R}^{N}} v \otimes v M_{\Theta} \mathrm{d} v=-\Theta \mathbb{I}, \\
J_{\lambda} & =\int_{\mathbb{R}^{N}} v \otimes \lambda \mathrm{d} v=-\int_{\mathbb{R}^{N}} v \otimes v \frac{1}{3}\left(\frac{v^{2}}{2}+(N+2) \Theta\right) M_{\Theta} \mathrm{d} v \\
& =-\frac{1}{3}(N+2)(1 / 2+1) \Theta^{2} \mathbb{I}=-\frac{N+2}{2} \Theta^{2} \mathbb{I},
\end{aligned}
$$

while for the heat flux we obtain

$$
\begin{aligned}
& q_{\chi}=\int_{\mathbb{R}^{N}} v \frac{v^{2}}{2} \otimes \chi \mathrm{d} v=-\frac{N+2}{2} \Theta^{2} \mathbb{I} \\
& q_{\lambda}=\int_{\mathbb{R}^{N}} v \frac{v^{2}}{2} \otimes \lambda \mathrm{d} v=-\frac{1}{3}\left(\frac{(N+4)(N+2)}{4}+\frac{(N+2)^{2}}{2}\right) \Theta^{3} \mathbb{I} .
\end{aligned}
$$

Finally, we are led to the following asymptotic behavior

$$
\begin{aligned}
J^{\epsilon} \underset{\epsilon \rightarrow 0}{\longrightarrow} J_{\chi} \cdot & \left(\nabla_{x} n-\left(u+\nabla_{x} \Phi\right) \frac{n}{\Theta}-\frac{N}{2} \frac{n}{\Theta} \nabla_{x} \Theta\right)+J_{\lambda} \cdot \frac{n}{\Theta^{2}} \nabla_{x} \Theta \\
& =-\Theta \nabla_{x} n+n\left(u+\nabla_{x} \Phi\right)+\frac{N}{2} n \nabla_{x} \Theta-\frac{N+2}{2} n \nabla_{x} \Theta \\
& =n\left(u+\nabla_{x} \Phi\right)-\nabla_{x}(n \Theta) .
\end{aligned}
$$


Similarly, we obtain

$$
\begin{aligned}
q^{\epsilon} \underset{\epsilon \rightarrow 0}{\longrightarrow} q_{\chi} \cdot\left(\nabla_{x} n-\left(u+\nabla_{x} \Phi\right) \frac{n}{\Theta}-\frac{N}{2} \frac{n}{\Theta} \nabla_{x} \Theta\right)+q_{\lambda} \cdot \frac{n}{\Theta^{2}} \nabla_{x} \Theta \\
=-\frac{N+2}{2}\left(\Theta^{2} \nabla_{x} n-n \Theta\left(u+\nabla_{x} \Phi\right)-\frac{N}{2} n \Theta \nabla_{x} \Theta\right) \\
-\frac{(N+4)(N+2)+2(N+2)^{2}}{12} n \Theta \nabla_{x} \Theta \\
=\frac{N+2}{2} n \Theta\left(u+\nabla_{x} \Phi\right)-\frac{N+2}{2} \Theta^{2} \nabla_{x} n-\frac{2(N+2)}{3} n \Theta \nabla_{x} \Theta .
\end{aligned}
$$

We summarize the result as follows.

Proposition 2. The fluctuation $r^{\epsilon}$ converges to

$$
r=-v M_{\Theta} \cdot\left(\nabla_{x} n-\left(u+\nabla_{x} \Phi\right) \frac{n}{\Theta}-\frac{N}{2} \frac{n}{\Theta} \nabla_{x} \Theta\right)-v M_{\Theta} \frac{1}{3}\left(\frac{v^{2}}{2}+(N+2) \Theta\right) \cdot \frac{n}{\Theta^{2}} \nabla_{x} \Theta,
$$

and the mass and heat fluxes have the following behavior

$$
\begin{aligned}
& J=n\left(u+\nabla_{x} \Phi\right)-\nabla_{x}(n \Theta), \\
& q=\frac{N+2}{2} n \Theta\left(u+\nabla_{x} \Phi\right)-\frac{N+2}{2} \Theta^{2} \nabla_{x} n-\frac{2(N+2)}{3} n \Theta \nabla_{x} \Theta .
\end{aligned}
$$

It allows to identify the limit in (13), and, of course, we recover in this way System (17).

\section{NUMERICAL SCHEME FOR THE LIMIT SYSTEM}

In the section we explore the limit system (17) from a numerical point of view. The space dimension is $N=1$ in the following, and the potential is gravitational: $\nabla_{x} \Phi=g$; System (17) reads

$$
\left\{\begin{array}{l}
\partial_{t} \rho+\partial_{x}(\rho u)=0 \\
\partial_{t} n+\partial_{x}(n u)=\partial_{x, x}^{2}(n \Theta)-g \partial_{x}(n) \\
\partial_{t}(\rho u)+\partial_{x}(\rho u \Theta u+p)=-\partial_{x}(n \Theta)+(n-\rho) g \\
\partial_{t}\left(\rho E+\frac{N}{2} n \Theta\right)+\partial_{x}\left((\rho E+p) u+\frac{3}{2} n \Theta u\right) \\
\quad=\frac{3}{2} \partial_{x}\left(\Theta^{2} \partial_{x} n\right)+2 \partial_{x}\left(n \Theta \partial_{x} \Theta\right)-\frac{5}{2} g \partial_{x}(n \Theta)+(n-\rho) u g+n g^{2}
\end{array}\right.
$$

The numerical scheme that we derived is based on the following form of the system: denoting $\tilde{p}=p+n \Theta$ and $\rho \tilde{E}=\rho E+n \Theta / 2$, the system can be recast as

$$
\left\{\begin{array}{l}
\partial_{t} \rho+\partial_{x}(\rho u)=0 \\
\partial_{t} n+\partial_{x}(n u)=\partial_{x, x}^{2}(n \Theta)-g \partial_{x}(n), \\
\partial_{t}(\rho u)+\partial_{x}(\rho u \otimes u+\tilde{p})=(n-\rho) g \\
\quad \partial_{t}(\rho \tilde{E})+\partial_{x}((\rho \tilde{E}+\tilde{p}) u) \\
\quad=\frac{3}{2} \partial_{x}\left(\Theta^{2} \partial_{x} n\right)+2 \partial_{x}\left(n \Theta \partial_{x} \Theta\right)-\frac{5}{2} g \partial_{x}(n \Theta)+(n-\rho) u g+n g^{2} .
\end{array}\right.
$$

Then the design of the scheme is based on a splitting strategy. Every time step is decomposed in 3 stages. 
- The first stage solves the system without the right-hand side above:

$$
\left\{\begin{array}{l}
\partial_{t} \rho+\partial_{x}(\rho u)=0 \\
\partial_{t} n+\partial_{x}(n u)=0 \\
\partial_{t}(\rho u)+\partial_{x}(\rho u \otimes u+\tilde{p})=0 \\
\partial_{t}(\rho \tilde{E})+\partial_{x}((\rho \tilde{E}+\tilde{p}) u)=0
\end{array}\right.
$$

Here we note the similarity with the classical Euler system. For this stage a standard Lagrange-remap scheme, as in [6], [7], is used. The stability of this stage relies on a hyperbolic-type condition on the time step: the time step of the whole time iteration is determined by this "hyperbolic" condition.

- The second stage solves the dissipative terms as well as the "transport" terms due to the gravity:

$$
\left\{\begin{array}{l}
\partial_{t} \rho=0 \\
\partial_{t} n=\partial_{x, x}^{2}(n \Theta)-g \partial_{x}(n) \\
\partial_{t}(\rho u)=0 \\
\partial_{t}(\rho \tilde{E})=\frac{3}{2} \partial_{x}\left(\Theta^{2} \partial_{x} n\right)+2 \partial_{x}\left(n \Theta \partial_{x} \Theta\right)-\frac{5}{2} g \partial_{x}(n \Theta)
\end{array}\right.
$$

This stage imposes a parabolic-type stability condition on the time step and is sub-cycled in the time step.

- the last stage solves the gravity terms:

$$
\left\{\begin{array}{l}
\partial_{t} \rho=0 \\
\partial_{t} n=0 \\
\partial_{t}(\rho u)=(n-\rho) g \\
\partial_{t}(\rho \tilde{E})=(n-\rho) u g+n g^{2}
\end{array}\right.
$$

This is done numerically with an explicit Euler scheme.

\section{NumericAl RESUlts}

The space domain is a bounded interval (namely $[0,4]$ in the following) and the chosen boundary conditions are wall boundary conditions: concerning the fluid, $u=0$ and $\partial_{x} p=0$. The boundary condition to be imposed on $\Theta$ is to be derived from the microscopic equations. The wall condition on the microscopic density $f$ reads $f(t, x, v)=f(t, x,-v)$ for every $v \in \mathbb{R}$, when $x$ belongs to the boundary. From (19) we have that $r$ obeys

$$
\mathscr{L}_{\Theta} r=v M_{\Theta}\left(\partial_{x} n-(u+g) \frac{n}{\Theta}-\frac{1}{2} \frac{n}{\Theta} \partial_{x} \Theta+\frac{v^{2} n}{2 \Theta^{2}} \partial_{x} \Theta\right) .
$$

We remark that, imposing that $r$ is an even function in the $v$ variable on the boundary, the left-hand side is an even function and the right-hand side is an odd function. Thus one has

$$
\partial_{x} n-(u+g) \frac{n}{\Theta}-\frac{1}{2} \frac{n}{\Theta} \partial_{x} \Theta+\frac{v^{2} n}{2 \Theta^{2}} \partial_{x} \Theta=0
$$

for every $v \in \mathbb{R}$. Thus $\partial_{x} \Theta=0$ and $\partial_{x} n=(u+g) n / \Theta=g n / \Theta$ on the boundary.

The first numerical result we propose is the case of an isentropic fluid with pressure law $p=\rho^{\gamma}(\gamma \geq 1$, namely here $\gamma=1.4)$. In this case the energy equation for the fluid is dropped. This allows to compare the results with those obtained for the microscopic (kinetic) system (for $\epsilon>0$ but small) in [4]. The test-case is one of those presented in this reference: this initial condition is at rest, with $u(0, x)=0, \rho(0, x)=1$ and $n(0, x)=0.5$. The 
final time is $T=2$. On figures $1,2,3$ we observe the strong similarity between the two numerical solutions. This similarity is improved when refining the mesh and having $\epsilon$ decrease.

The second series of numerical results are obtained with the same initial conditions, but we want here to analyze the effect of temperature. We thus compare the results of the limit system for an isentropic gas and for an ideal gas. On this first and preliminary result, we see that the thermal effect is not negligible. Figure 7 shows that the temperature has strong variations in the space variable. The consequences concerning the fluid density are particularly important, inducing a high fluid density region on the left-hand side (which can be considered as the bottom, since gravity makes the fluid go leftwards) of the domain. There is a lot of energy on the left-hand side, because of the fluid "falling down" with a high velocity, as we can see in Figures 4, 6 and 7. On the right-hand side, on the contrary, since the particles are going rightwards slowly because of the buoyancy effect, there is very little energy.

\section{REFERENCES}

[1] C. Baranger, G. Baudin, L. Boudin, B. Després, F. Lagoutière, E. Lapébie and T. Takahashi. Liquid jet generation and breakup. In Numerical Methods for Hyperbolic and Kinetic Equations, S. Cordier, Th. Goudon, M. Gutnic, E. Sonnendrücker Eds., IRMA Lectures in Mathematics and Theoretical Physics, vol. 7, EMS Publ. House, 2005.

[2] C. Baranger, L. Boudin, P.-E. Jabin and S. Mancini. A modeling of biospray for the upper airways. ESAIM:Proc, 14:41-47, 2005.

[3] J.-A. Carrillo and Th. Goudon. Stability and asymptotics analysis of a fluid-particles interaction model. Comm. PDE, 31:1349$1379,2006$.

[4] J.-A. Carrillo, Th. Goudon and P. Lafitte. Simulation of Fluid \& Particles Flows: Asymptotic Preserving Schemes for Bubbling and Flowing Regimes. J. Comput. Phys., 227(16):7929-7951, 2008.

[5] J.-A. Carrillo, Th. Goudon, P. Lafitte, and F. Vecil. Numerical schemes of diffusion asymptotics and moment closures for kinetic equations. J. Sci. Comput., 36(1):113-149,2008.

[6] B. Després, Inégalité entropique pour un solveur conservatif du système de la dynamique des gaz en coordonnées de Lagrange. C. R. Acad. Sci. Paris Sér. I Math. 324 (1997) no 11, p. 1301-1306.

[7] G. Gallice, Schémas de type Godunov entropiques et positifs préservant les discontinuités de contact. C. R. Acad. Sci. Paris Sér. I Math. 331 (2000) no 2, p. 149-152.

[8] Th. Goudon, P. Lafitte. Splitting schemes for the simulation of non equilibrium radiative flows. Preprint, 2007.

[9] Th. Goudon, P.-E. Jabin and A. Vasseur. Hydrodynamic limit for the Vlasov-Navier-Stokes equations. I. Light particles regime. Indiana Univ. Math. J., 53(6):1495-1515, 2004.

[10] Th. Goudon, P.-E. Jabin and A. Vasseur. Hydrodynamic limit for the Vlasov-Navier-Stokes equations. II. Fine particles regime. Indiana Univ. Math. J., 53(6):1517-1536, 2004.

[11] M. Massot and Ph. Villedieu. Modélisation multi-fluide eulérienne pour la simulation de brouillards denses polydispersés. $C$. R. Acad. Sci. Paris Sér. I Math., 332(9):869-874, 2001.

[12] J. Mathiaud. Etude de systèmes de type gaz-particules. PhD thesis, ENS Cachan, Sept. 2006.

[13] B. Sportisse. Modélisation et simulation de la pollution atmosphérique. Habilitation à Diriger les Recherches, Sciences de l'Univers, Université Pierre et Marie Curie, June 2007.

[14] R. F. Streater. The Soret and Dufour effects in statistical dynamics. Preprint 1998 (arXiv:math-ph/9910043v1).

[15] F.A. Williams. Combustion theory. Benjamin Cummings, 1985. 


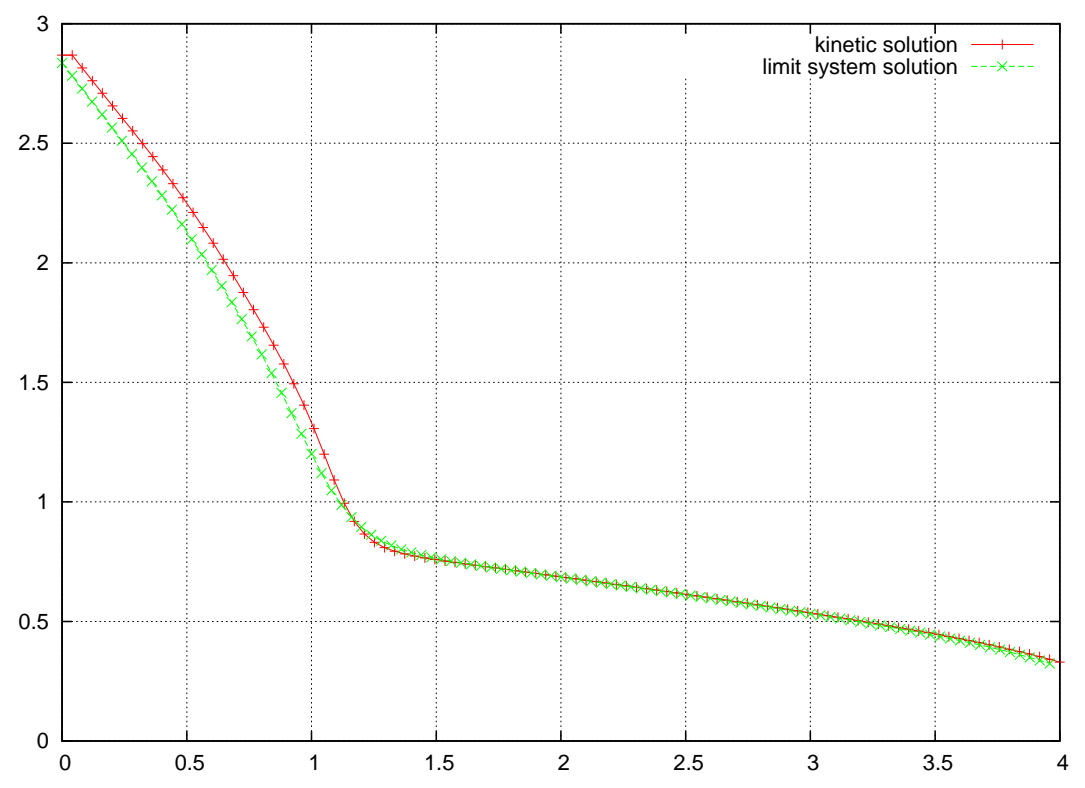

FIGURE 1. Fluid density: comparison of the limit solution and the solution with $\epsilon=0.1$.

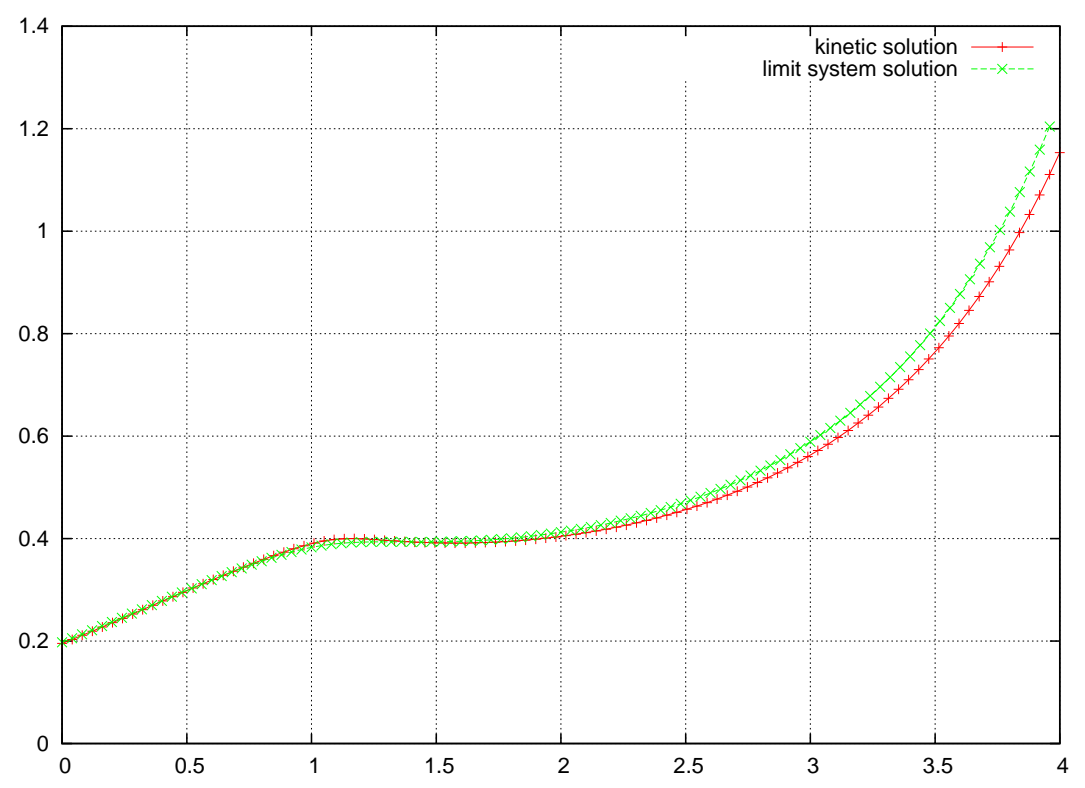

Figure 2. Particles macroscopic density: comparison of the limit solution and the solution with $\epsilon=0.1$. 


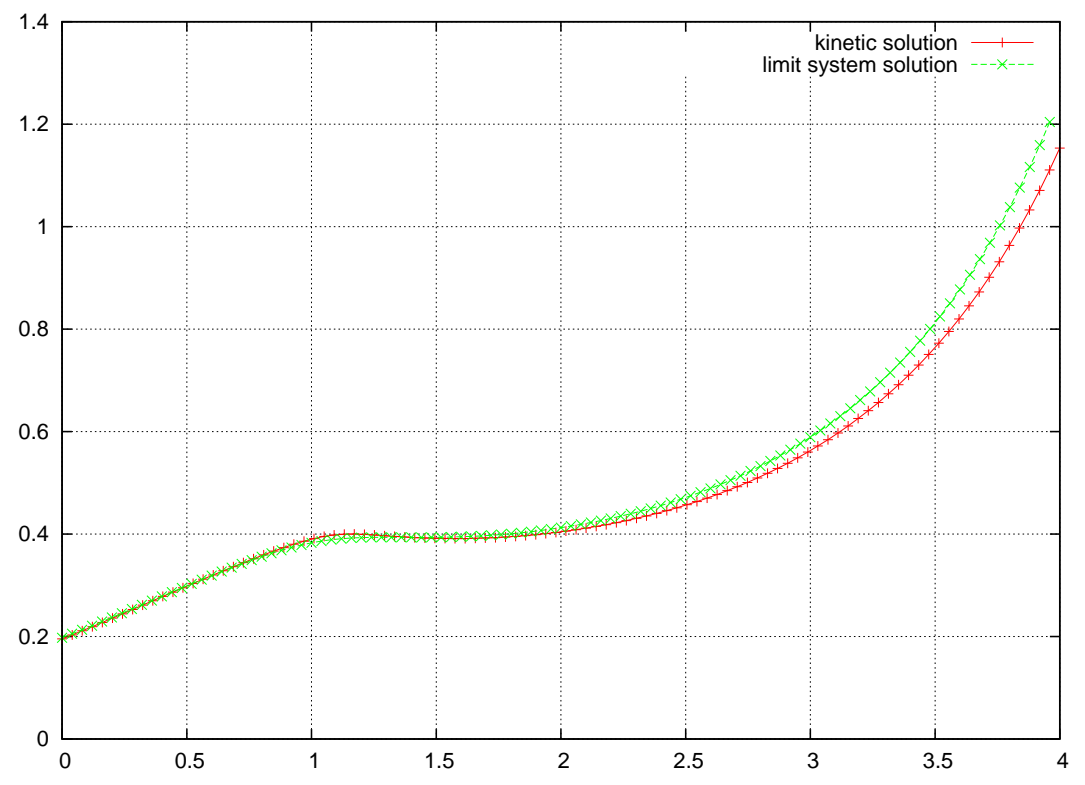

FIGURE 3. Fluid velocity: comparison of the limit solution and the solution with $\epsilon=0.1$.

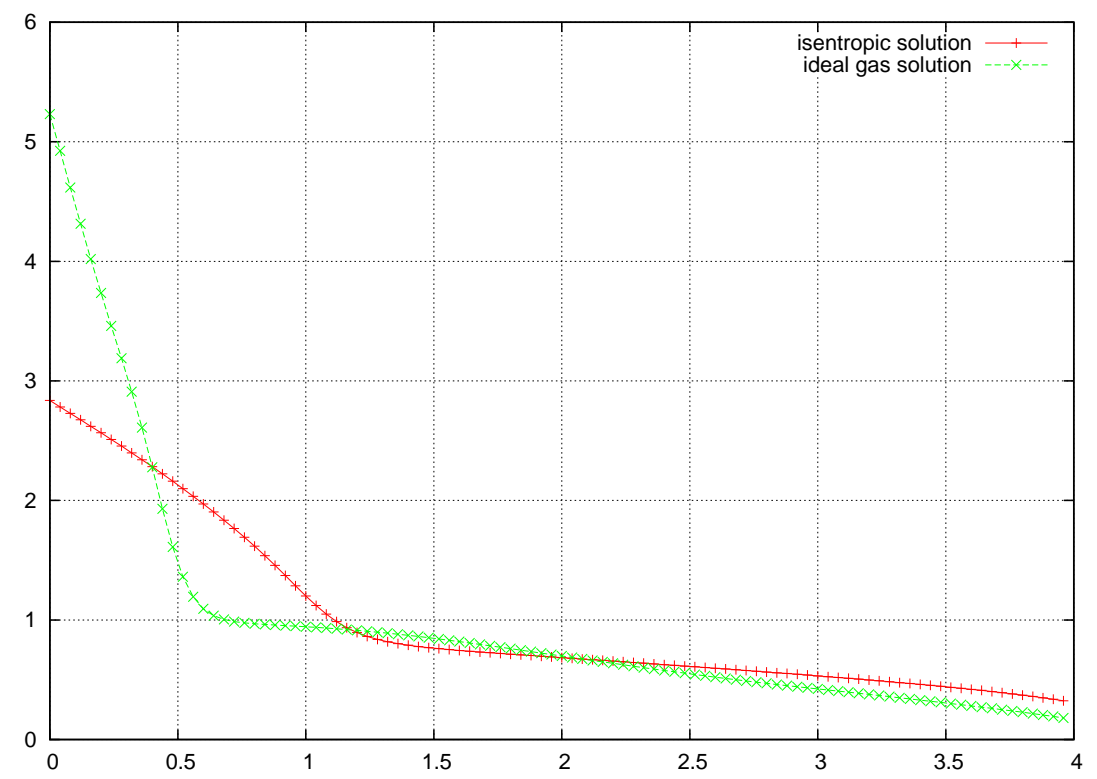

FIGURE 4. Fluid density: comparison of the isentropic and the ideal gas solutions. 


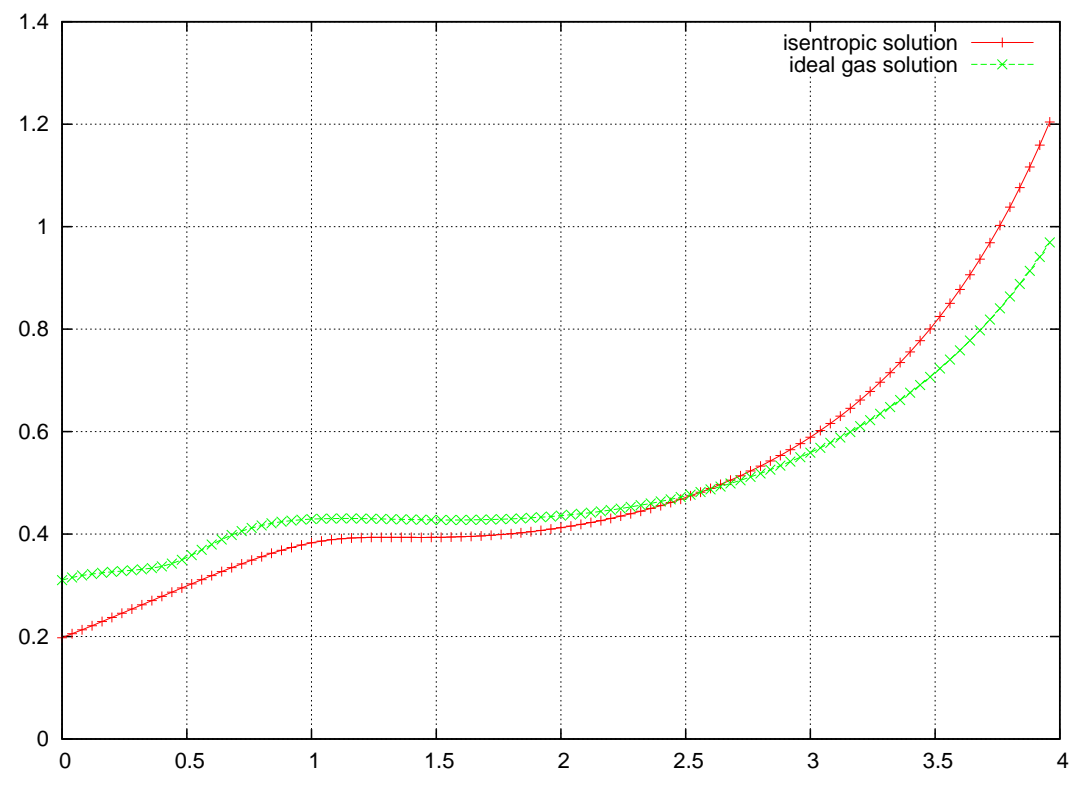

Figure 5. Particles macroscopic density: comparison of the isentropic and the ideal gas solutions.

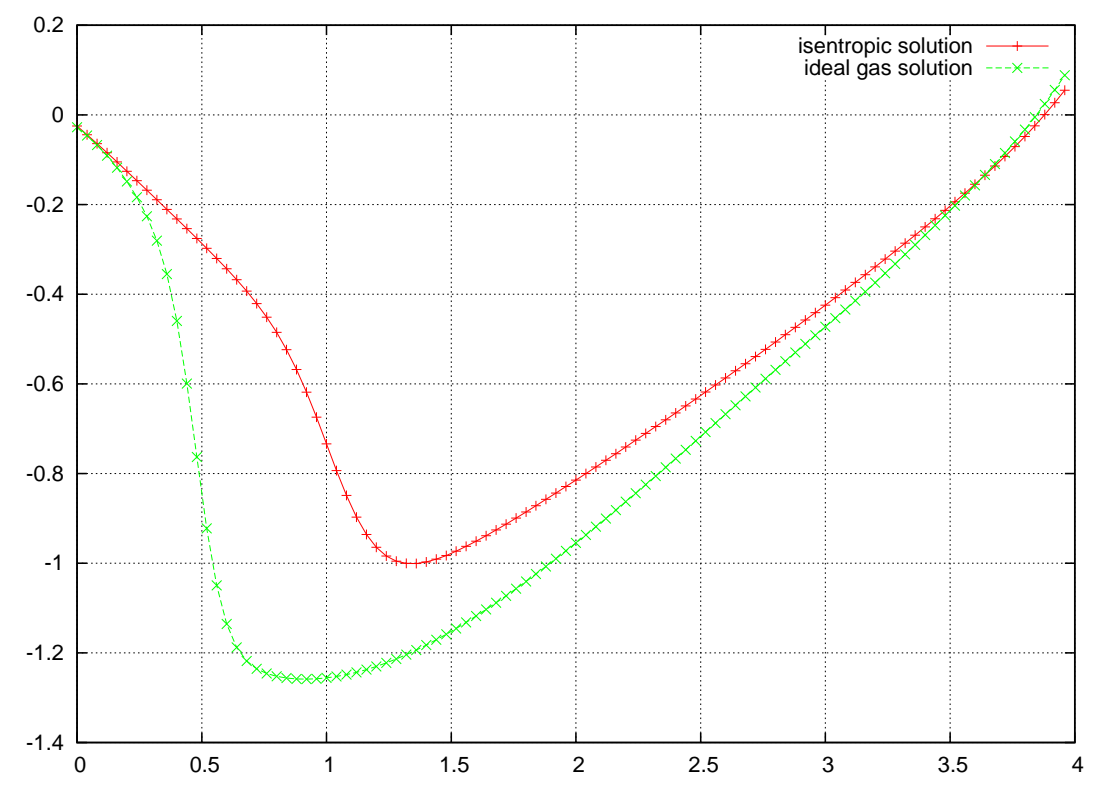

FIGURE 6. Fluid velocity: comparison of the isentropic and the ideal gas solutions. 


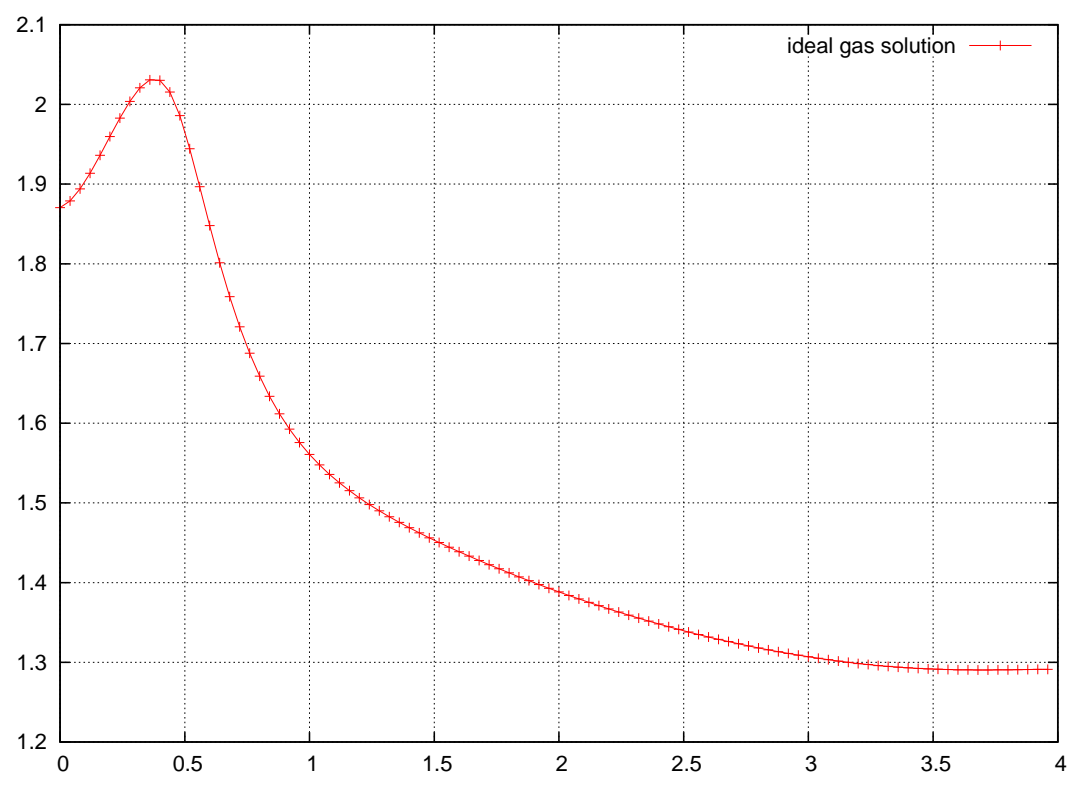

FiguRE 7. Temperature (ideal gas pressure law). 